\title{
الحريات العامة في الدولة الإسلامية
}

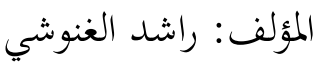 \\ الناشر: مركز دراسات الوحدة العربية، بيروت، الطبعة الأولى، 1993، 382 صفحة.
}

مراجعة: الفاتح عبد الله عبد السلام²

ليس من سرف القول إن الفكر الإسلامي المعاصر يشهد دورة معرفية نشطة تسعى نهو توجيه ذلك الفكر في ابتحاه مشكلات الحضارة وعمليات النهوض الحضاري للأمة الإسلامية. وتهدف هذه الدورة المعرفية إلى تطهير الفكر الإسلامي من كل رواسب التخلف والانطاط رقيا إلى التجديد والاجتهاد ليعود ذلك الفكر أصيلا مستجيبا لتحديات العصر متجاوبا قضاياه في إطار المرجعية العليا للوحي الإلهي.

وتتجلى هذه الصحوة في الثورة المفهومية في مجالات حياتنا الفكرية، ولا غرابة في ذلك إذا ما نظرنا إلى التجديد الإسلامي بوصفه ظاهرة تاريخية دورية كلما اعترى المسلمين ذبول في دوافع الإيمان وخمول في الفكر وبمود في الحركة واستفزهم التحدي الخارجي.

وقد بتحاوز توسع هذه الظاهرة وامتدادها دائرة النشاط الإسلامي المنظم حتى غدت تيار فكريا متندا وظاهرة اجتماعية واسعة وشعورا قويا بضرورة التحرر من سلطان قيم الفكر وأنماط الحياة الغربية وبالأوبة إلى أصول الانتماء الإسلامي، والسعي لتمكين قيم الدين في واقع الحياة مما جعلها تتحول إلى حركة تجديد شاملة لكيان الأمة الإسلامية ولقدرها الجماعية على الفعل الحضاري.

من الجدليات الشائكة والساخنة والمتجددة التي شغلت الفكر السياسي الإسلامي الحديث مسألة الديمقراطية والحريات العامة في العقل الغربي والعقل الإسلامي. وتعد هذه الإشكالية، دون مبالغة، قضية القضايا في

\footnotetext{
* أستاذ مساعد بقسم العلوم السياسية في الجامعة الإسلامية العالمية - ماليزيا، دكتوراه في العلوم السياسية من جامعة نورث ويسترن في الولايات المتحدة الأمريكية.
} 
الفكر الإسلامي الحركي الحديث. وإذا كان الاسلام بوصفه نظاماً شاملا ومتكاملا واجه في كثير من بلاد المسلمين ضغوطا شديدة الوطأة محورها أن الإسلام لا يملك القدرة على تنتظم الحياة السياسية والاجتماعية ولا ضمان فيه للحريات العامة والفردية وحقوق الأقليات، وبالتالي لا يملك تصورا محددا للدولة، فإن الديمقراطية الليبرالية الغربية قد قدمت بالمقابل، على أها الضد النقيض للإسلام. ليس ذلك فحسب. بل كلمة الديمقراطية الغربية تفرض على أها النظام الأمثل القابل للتطبيق في مواجهة شعارات الإسلاميين التي تتهم بأها ستثير المشاعر والعواطف فحسب، ولا تصلح منهاجا لتنظيم الحياة في مختلف جوانبها!

لقد أدت هذه الإشكالية إلى انقسام الآراء والاتحاهات بصورة كبيرة نتيجة التنوع الأيديولوجي والسياسي في العالم الإسلامي. وهذا التعدد قد حصل حتى في داخل التيار الواحد كالتيار الإسلامي باتحاهاته السياسية والحركية المختلفة، فنحن نجد مثلا، توجهات رافضة لفكرة الديمقراطية، إلا أهما توجهات لا تمثل كتلة واحدة، فهي تتفاوت في تسويغها الرفض أو التحفظ حيال فكرة الديمقراطية أو في قبولها المشروط أو المقيد لها. وفي هذا الإطار نجد توجها نظريا رافضا للفكرة جملة وتفصيلا بناء على تصور معين لها يظنه ثابتا وكامنا في الفكرة الديمقراطية ذاتا، وأها ليست من الاسلام ولا تلتقي مع قيمه ومبادئه وأن العلاقة بينهما علاقة تعارض وتناقض. 1

وهناك توجه آخر يرى أن المسلمين ليسوا بحاجة إلى الديمقراطية لأفم يملكون فكرة بديلة هي الشورى، بل يذهب بعضهم على القول بأن مفهوم الشورى أرقى وانضج وأكثر شمولا من مفهوم الديمقراطية، إذ مصدرها وأصلها الوحي الإلهي الذي جاء بمنهج متكامل لحياة الإنسان، في حين أن الديمقراطية، مفهوما ومنهجا، وقيما، مجرد نتاج للتجربة الحضارية الغربية.

وهناك تةجه ثالث يرفض فكرة الديمقراطية لانتمائها بصلة القرابة الفلسفية مع فكرة "العلمانية". والعلمانية مرفوضة لتناقضها مع فكرة الشريعة وحاكميتها ولاستبعادها الدين من تنظيم الشؤون الاجتماعية.

1 انظر على سبيل المثال لا الحصر: كاظم الحائري: أساس الحكومة الإسلامية، بيروت: الدار الإسلامية، 1979م. ويمكن الاستئناس في هذا الشأن بكتابات "حزب التحرير الإسلامي" في موقفه من الديمقراطية.

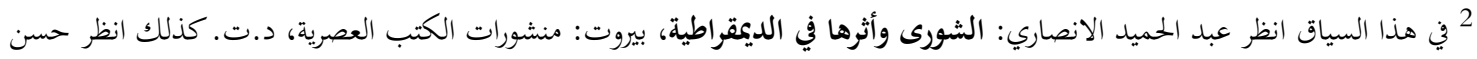

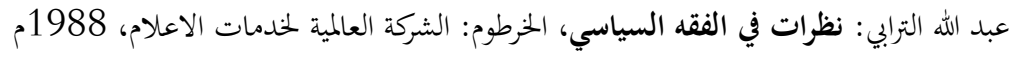


وهناك تيار رابع يسوغ رفضه للديمقراطية بسبب بعض الإشكاليات المرتبطة بجملة من آلياته مثل الحزبية والدعاية الانتخابية والمعارضة الشكلية والأغلبية والأقلية ومبدأ النيابة عن الشعب، وهي أمور يرى أها تفتقر إلى المحتوى الموضوعي. ومن ثم فهذا التيار يرفض الممارسات الشكلية والمظهرية لبعض النظم السياسية باسم الديمقراطية وبأشكال برلمانية ودستورية زائفة. 3

وهناك، أخيراً ، تيار يبني رفضه لفكرة الديمقراطية على اعتبارات خلقية، مثل كون مفهوم الحرية الملازم لفكرة الديمقراطية في الثقافة الغربية ينطوي على نزوع إباحي وعدم انضباط خلقي، مما يتناف مع مبادئ الشريعة الإسلامية وفلسفتها الخلقية.

وتلتقي كل هذه المواقف الرافضة للديمقراطية في أن الأخذ بها يعني استبدال التشريع الإلهي الذي ورد به الوحي بتشريع وضعي محدود بإطار خبرة حضارية معينة.

والملاحظة الجديرة بالتسجيل هنا أن التيارات الرافضة، رفضا باتا، للديمقراطية باسم الإسلام، تمثل بجموعات صغيرة أبرزها "السلفيون"، ولعل سبب موقفهم هذا أفم لم يمارسوا العمل السياسي إلا مؤخراً. وهم كذلك حرفيون في منهجهم الفكري لأغم آثروا الانكفاء على القديم ولم ويواكبوا حركة التطور الفكري والاجتماعي التي شهدهما مجتمعات المسلمين.4

وفي مقابل التيارات الفكرية الرافضة لفكرة الديمقراطية، هناك تيار إسلامي عريض حاول مفكروه ومنظوره تأصيل فكرة الديمقراطية والتأسيس النظري لها في السياق الفكري الإسلامي. ويحمد لرموز هذا التيار ومنظريه أفم بطرحهم هذا، يتجاوزون عقلية المقابلة والمناظرة بين الإسلام والديمقراطية التي تستغرقها سطوح القضايا وشكلياها ليبلوروا فكراً سياسيا يستوعب جوهر فكرة الديمقراطية ويبرز أبعادها الإسلامية، أو إن شئنا قلنا يبرزون كيف أن الإسلام يحتوي على مكون "ديمقراطي" أصيل.5

$$
\begin{aligned}
& 3 \text { انظر مثلا خالد محمد خالد: الديمقراطية.... ابدا، القاهرة: مؤسسة الخانجي، 1958م. } \\
& 4 \text { محمد قطب: مذاهب فكرية معاصرة، بيروت: دار الشروق، 1983 1983م. }
\end{aligned}
$$

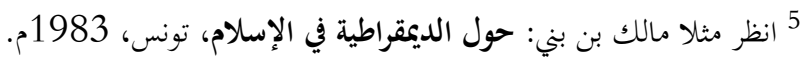

$$
\begin{aligned}
& \text { وعباس محمود العقاد: الديمقراطية في الإسلام، القاهرة: دار المعارف، د ت. }
\end{aligned}
$$


وحتى وقت قريب كان الاستقراء السريع لمدى حضور فكرة الديمقراطية، بوصفها نظرية ومنهجا، في الأعمال الفكرية الإسلامية منذ حركة الإصلاح الإسلامي في النصف الثاني للقرن الماضي، يكشف لنا أن الديمقراطية وإن كان لها حضور في العمل الفكري الإسلامي إلا أنه حضور محدود وعرضي. ويكشف هذا الاستقراء عن إثكالية منهجية في الفكر الإسلامي من حيث معالجة القضايا الفكرية المعاصرة؛ وجوهر هذه الإشكالية المنهجية أن الفكر الإسلامي كان أقرب إلى الدفاع عن الذات ومحاولة إبراز عناصر التشابه والتماثل بين المبادىء الإسلامية والمبادىء التي تقوم عليها الحضارة الغربية، دون ارتقاء منهجيته إلى المستوى الذي يؤهلها لبلورة وصياغة فكر إسلامي يعالج الاشكاليات النظرية والعملية التي تواجه بجتمعات المسلمين بصورة علمية أصيلة بعيدا عن الاعتذارية أو ردود الفعل.

هذا ويمكن أن نلاحظ أن مسألة الديمقراطية تُطرح منذ بداية عهد التسعينيات من هذا القرن، بوتيرة

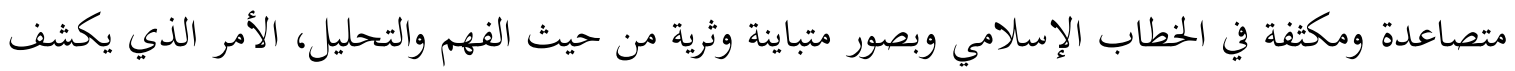
عن تحول في الرؤية السياسية وتغير في النظرية الفكرية السياسية عند المفكرين الإسلاميين المحدثين. وبعبارة أخرى، إن الديمقراطية أخضعت للتشريح من قبل المفكرين الإسلاميين بمدف معرفة مكوناتها وأبعادها وآفاق تكييفها وإمكانياته. وقد كان من نتائج ذلك التسليم والقبول بمشروعية بعض تلك المكونات وليس كلها، مثل مبدأ تداول السلطة بشكل سلمي، ومبدأ التعددية الحزبية والتعايش السلمي بين الجماعات على اختلاف طروحاةًا واجتهاداها، وحقوق الإنسان ورعايتها، والفصل بين السلطات، وما شابه ذلك مما يتعلق بإدارة الحياة والفعل السياسي. وهذا أمر بالغ الدلالة، إذ إنه يكشف عن تحول منهجي معريف في الوقت نفسه يشهده الفكر الإسلامي المعاصر الذي كانت تغلب عليه الاطلاقية واللاتاريخية. 6 وفي هذا السياق من تطور الفكر الإسلامي الحديث تأتي دراسة الشيخ راشد الغنوشي التي خصصها لبحث "قضية الحرية" وإخضاعها للمناقشة الفكرية والعلمية. ويلاحظ هنا أن انخياز الكاتب للموقف الإسلامي المؤيد للحرية ضد الاستبداد والانطلاق ضد الجمود كان انخيازا حاسما لا يداخله أي تردد.

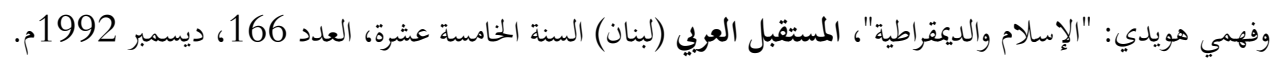

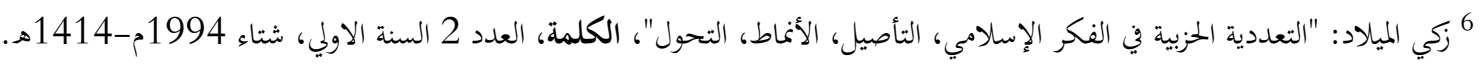

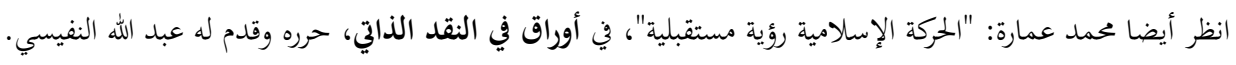


والكاتب في كلياته يطح عددا من الأسئلة الفكرية المحورية: هل هناك مفهوم لحقوق الإنسان في الإسلام؟ هل هناك أساس لمفهوم الدولة في الإسلام، وإن وجد فما الفرق بينه وبين مفاهيم الدولة في الثقافة الغربية الحديثة؟ ما الأبعاد السياسية والاقتصادية والتربوية للشورى؟ وما ضمانات الحركة في الدولة الإسلامية ضد الجور. تلك هي جملة القضايا المحورية التي عرض لها الكتاب. ولنعرض الآن بشيء من التفصيل لأهم مقولات المؤلف.

أفرد الأستاذ الغنوشي المبحث الأول من الكتاب لدراسة حقوق الإنسان وحرياته في الإسلام. فهو يرى أن الحرية في الإسلام قيمة أساسية أصيلة، وأها ليست مجرد إباحة، بل هي واجب وكدح متواصل لتجاوز الضرورة عبر مجاهدة النفس لحملها على معالي الأمور وبجاهدة قوى الشر والجهالة لإعلاء كملة الحق والعدل. ويذهب المؤلف إلى أن حقوق الإنسان في الإسلام تنطلق من مبدأ عقدي أساسي مفاده أن الإنسان يحمل في ذاته تكريما إلهيا أزليا وأنه، بناء على ذلك، مستخلف من الله في الكون، الأمر الذي يخوله حقوقا لا سلطان لأحد عليها من البشر. وبالمقارنة والمقابلة بين حقوق الإنسان في الإسلام وفي الإعلانات الغربية لحقوق الإنسان، يوضح المؤلف أن مجال اللقاء بينهما جد رحب، وأن الاختلافات يسيرة، وتشكل الاستثناء لا القاعدة، وأن الاختلاف لا يكمن في المضامين الجوهرية وإنما في الأسس الفلسفية والدوافع والغايات والمقاصد. ففي رأيه، يؤخذ على الإعلان العالمي لحقوق الإنسان، مثلا، استناده إلى أسس فلسفية غامضة مثل القانون الطبيعي - وهو مفهوم مبهم وغير محدد- الأمر الذي يحرم تلك الحقوق من العمق الروحي والخلقي ومن البواعث القوية للالتزام هما ورعايتها. وفي مقابل ذلك، نجدها في الإسلام جزءا لا يتجزأ من الشريعة ومقاصدها بل ومن البناء العقدي الإسلامي مله مما يكسبها صفة الواجب وقوة الإلزام والدوام والمبدئية.

وقد عالج المؤلف موضوع الإطار العام لحقوق الإنسان في خمسة مباحث فرعية: أفرد أولها لحرية الاعتقاد مركزا ما يمكن أن يرد على هذا الحق من قيود، فناقش موضوع الردة وأزال التناقض الذي يمكن أن يتوهم بينه وبين مبدأ الحرية. ثم عرض للآثار المترتبة على حرية الاعتقاد مثل حرية التعبير والمناقشة وممارسة الشعائر والحرية الفكرية، وأبان كيف أن الإسلام أحاطها بسياج سميك من الضمانات والحماية. ثم تناول حرية الذات أو ما 
أسماه" حق التكريم الإلهي "، أي سلامة الشخصية الإنسانية وحمايتها حيث تعرض بالتمحيص والغربلة لموضوع العنف والتعذيب وأساليب الإكراه وانتهى إلى الحكم بأها مخظورة حظرا مطلقاً.

ثم عرج على موضوع الحقوق الاقتصادية فأثبت حق التملك على أساس العمل وحق العامل في التمتع بثمار عمله والنظر إلى الملكية بصفتها وظيفة اجتماعية يمارسها الفرد تحت رقابة ضميره الديني وسلطة المجتمع في إطار مصلحة الجماعة، فإذا أساء الفرد التصرف تدخل المجتمع صاحب الحق نيابة عن المالك الأصلي، الله سبحانه وتعالى أما مسألة الحقوق الاجتماعية، فقد أكد الباحث أن العمل واجب ديني وأن في مال الأغنياء حقا معلوما لمن لا يملك، وانتهى إلى أنه لا حرمة لمال طالما في المجتمع محتاج معدم قد تعرض لجملة من الحقوق الاجتماعية السياسية التي رعاها الإسلام مثل حق التعليم الإلزامي والحق في الرعاية الصحية والحق في

$$
\text { السكن والكساء وإقامة الأسرة. }
$$

والواقع أن مفهوم "العدالة الاجتماعية" أحد أبرز المفاهيم التي أثارها الفكر الاجتماعي السياسي الإسلامي المعاصر. وكان سيد قطب رائد توجيه الفكر الإسلامي إلى هذا الموضوع. ولعل ذلك يرجع إلى عاملين: الأول: يتمثل في تفاقم أحوال الفقر وسوء توزيع الثروة في المجتمعات الإسلامية، والثاني: الضغط السياسي الذي مارسته الحركات الاجتماعية السياسية "الجماعية" أو "الشيوعية" في مطلع الخمسينات من هذا القرن غير أن كتاب سيد قطب لم يقدم إلا وصفا عاما للمفهوم ومبادئ عامة تحكمه. 7 وقد تابع هذه الأفكار مفكرون إسلاميون آخرون كان في مقدمتهم الداعية السوري مصطفى السباعي الذي ضمن أفكاره كتابا لا يخرج في جملته عن كتاب سيد قطب، نشره في عام 1959 بعنوان "اشتراكية الإسلام."

أما المبحث الرئيسي الثاني لكتاب "الحريات العامة" فقد خصصه المؤلف لقضية الحريات السياسية مقارنا بين المبادئ الأساسية للديمقراطية الغربية ونظام الحكم الإسلامي أو ما نعته "بالديمقراطية الإسلامية". وفي معالجته لمفهوم الدولة في الفكر الديمقراطي الغربي حاول المؤلف إبراز ما في هذا المفهوم من تناقض وغموض وأخطار كامنة بسبب الأساس الفلسفي المادي والأساس السياسي القومي الذي يسنده، والذي كان من نتائجه الحروب القومية والاقتصادية وانتشار الفقر والأوبئة الفتاكة وتدهور أحوال البيئة وتطور أسلحة الدمار الشامل 
على حساب الخدمات الإنسانية وتنمية الموارد وحماية البيئة. وقد أبان الكاتب أن النظام الديمقراطي الغربي يبقى في بحلياته وتطبيقاته بعيدا عن مثاله لأنه وإن تساوت في إطاره أصوات المواطنين نظريا، إلا أن انقسام المجتمع إلى طبقة أصحاب الاحتكارات الكبرى للمال ووسائل الإعلام ومأجورين وعاطلين يمثلون الأغلبية، كل ذلك طبع الديمقراطية الغربية بميسم الزيف والتناقض. ويبقى الخلل الرئيسي في هذا النظام كامنا في مضامينه الفلسفية المادي أي في "علمانيته" بما في ذلك تجليها الأكبر المتمثل في استبعاد الوحي والدين من تنظيم شؤون لمجتمع، و تأليه الإنسان بالتنكر للمطلق الحقيقي، الله سبحانه وتعالى خالق الكون ومدبره. ومن خلال دراسته للمبادئ الأساسية للحكم الإسلامي أو الديمقراطية الإسلامية، انتهى المؤلف إلى أن مفهوم الدولة أصيل في مبادئ الإسلام وفكره السياسي، وأن السلطة حاجة طبيعية وضرورة اجتماعية ومقتضى ديني لإقامة الدين، وأن السلطان المتجسد فيها يخول من الله للجماعة. ولأن الجماعة لا يمكن عمليا - أن تدبر السلطة مباشرة، تحتم إيكال هذه السلطة، بمقتضى عقد أو "بيعة"، لمن يقوم بها وفق القانون أو النص الذي أساسه الكتاب والسنة من جهة، والشورى ورقابة الشعب في مستوى التشريع والتنفيذ من جهة أخرى. - nم.

وقد تعرض المؤلف بالتفصيل لما اشترط من شروط لأعضاء الهيئة الشورية ووظيفتهم، وأظهر الاختلاف الجوهري بين مجلس الشورى والمؤسسة البرلمانية الغربية، إذ أن الهدف من الشورى ليس مجرد معرفة ابتحاه الأغلبية وإنما الإجماع أو ما يقرب منه. وإلى جانب الشورى في بعدها التشريعي، تحدث المؤلف عن أبعاد أخرى للشورى السياسية والشورى أو الحرية في الميدان الاقتصادي والشورى الثقافية التربوية، حيث ذهب إلى أن هذه الأبعاد لا تختلف، في جوانب عديدة منها وفي كثير من عناصرها، عن النموذج الليبرالي الغربي، إلا

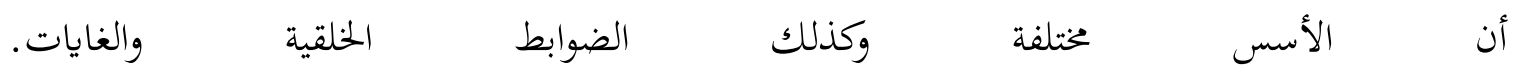
هذا وقد خص المؤلف المبحث الرئيس الثالث والأخير لدراسة ضمانات عدم الجور في الدولة الإسلامية. وهذه القضية من القضايا الرئيسة التي اهتم بها الفكر الإسلامي منذ زمن غير يسير. فرفاعة رافع الطهطاوي يرى أن "الحرية" عند الغربيين هي عين ما يسمى في الفكر الإسلامي بالعدل والإنصاف ويربط صراحة بين الحرية والعدل من ناحية وعمارة البلدان وكثرة معارف وتراكم الغنى وراحة القلوب، أي تفشي الأمن، من 
ناحية أخرى مذكراً في ذلك بمقولة ابن خلدون من أن العدل أساس العمران. 8 وقد تبعه في ذلك خير الدين التونسي الذي لا يختلف عنه كثيرا حيث حاول أن يقيس على المثال الأوروبي القائم على الأخذ بالتظيمات الدنيوية التي رأى أنه يكمن فيها تحديداً سر التمدن الغربي وحسن السياسة عند الأوروبيين مما ترتب عليه توسيع دائرة العلوم والمعرفة وتطوير مصادر الثروة من التجارة والزراعة والصناعة. بل إن المصلح خير الدين التونسي يختزل كل المسألة في القول إن "التنظيمات المؤسسة على العدل والحرية هي أساس ما أحرزه الأوروبيون من قوة وثراء ونماء ورفاهية. 9 وتابع ابن أبي الضياف، المؤرخ التونسي لعهد البايات، الحملة على لئل الجور والظلم وأعاد إلى الأذهان كلمة ابن خلدون الخالدة "إن الظلم مؤذن بفساد العمران."10ويعد المفكر السوري عبد الرحمن الكواكبي من ثلة المفكرين المناهضين للاستبداد والحكم المطلق حيث يركز اهتمامه على "تشريح جثة الاستبداد" وبيان علاقة هذا الداء بتقهقر الأمة وإعاقة حركتها الحضارية. وربما كان للكواكبي فضل تنبيهنا إلى أن القرآن الكريم نفسه مشحون بتعاليم إماتة الاستبداد وإحياء قيم العدل والإنصاف. 10 لقد طور الغنوشي أفكار هؤلاء المفكرين وزاد عليها. فهو يرى أن الدولة في النظام الماركسي الشيوعي ما هي إلا أداة لقمع الخصوم وتحقيق أهداف القلة وإشباع حاجاتا. أما في الديمقراطية الغربية وعلى الرغم مما تتوفر عليه من أجهزة الرقابة، فإن قيام الدولة على الفلسفة المادية جعل لقيم الربح واللذة والكبرياء القومي السلطان الأعلى على مؤسساةًا وأعطى ذوي النفوذ المادي سلطانا عظيما على عقول الناس وحياقم ومصائرهم. أما الدولة الإسلامية، فإن هدفها الأسمى، في رأي الغنوشي، إتاحة مناخات الحرية والعدالة والتطهر والترقي الروحي والمادي أمام أوسع قطاع من المواطنين. ثم يطرح المؤلف مجموعة من الضمانات تحول دون انحراف الدولة الإسلامية وترديها في الجور، خاصة وقد حدث ذلك تاريخيا. ومن الضوابط الأساسية التي يقترحها المؤلف النظر، أولاً: إلى أن المشروعية العليا في الدولة إنما هي لله سبحانه وتعالى من خلال شريعته، وأن الأمة هي المستخلفة عن الله وليس فردا أو مؤسسة أو جماعة بعينها، الأمر الذي يضع قيودا

8 رفاعة رافع الطهطاوي: الاعمال الكاملة لرفاعة رافع الطهطاوي، دراسة وتحقيق محمد عمارة، بيروت: المؤسسة العربية للدراسات والنشر،

9 خير الدين التونسي: أقوم المسالك في معرفة أحوال الممالك، تحقيق منصف الشنوفي، تونس: الدار التونسية للنشر، 1972م.

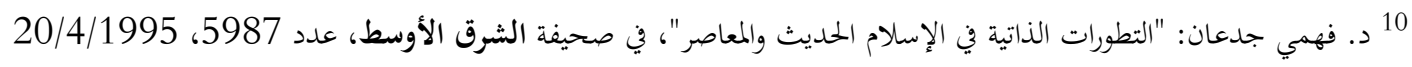

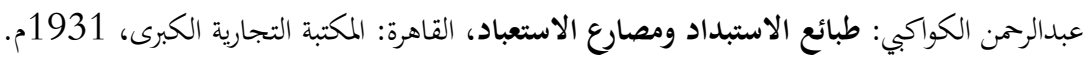


على سلطان الدولة التشريعي والتنفيذي. ثانياً: اعتبار عقد النيابة عقد وكالة خاص، الأمر الذي يجعل النائب أو عضو مجلس الشورى تحت مجهر رقابة الناخبين بشكل دائم. ثالثاً: اشترط عدم احتجاب الحكام في أبراج عاجية عن الشعب مع منع جمعهم بين سلطتي المال والحكم. رابعاً: إقامة نظام اقتصادي يضمن عدم تركيز الثروة ويسهّل توزيعها "وتكثير" عدد المالكين. خامساً: إقامة نظام اجتماعي يؤكد قيمة العمل ويعترف بالتملك وحق الفقير في مال الغني. سادساً: إقامة نظام تربوي يشيع المعرفة وييسر وسائلها ويرفع سلطان الدولة عن عقول الناس وأرواحهم. سابعاً: إقامة نظام تعدد الأحزاب بضوابط معينة وضمان عدم اشتغال الأحزاب بالصراعات الهامشية على حساب المصلحة العليا العامة. ثامنا وأخيرا: إقامة نظام إداري للحكم المحلي يسحب معظم صلاحية الحكومة المركزية ليضعها في يد الشورى الشعبية.

وإلى جانب الضوابط أو الضمانات السابقة الذكر، يشير المؤلف إلى مبدأ فصل السلطات بوصفه ضمانا من ضمانات عدم الجور، مؤكدا على استقلالية القضاء ونفاذ أحكامه، ومضيفا أن الموقف الإسلامي يحتمل الفصل والتعاون أو الاندماج في شأن العلاقة بين سلطة التشريع والتنفيذ. بيد أننا نجده يرجح الفصل غير المغلظ وأخيراً: يتناول المؤلف ما حدث من جور في تاريخ الدولة الإسلامية على الرغم من أن الإسلام قرن بين الاستبداد والظلم وبين التوحيد والعدل. وفي هذا السياق يْْصل الغنوشي أو يميز بين النظرية (الشريعة) والتاريخ الإسلامي من حيث أنه يمثل الجانب التطبيقي الذي هو فعل المكلفين. فما حدث من جور ليس، في رأيه، من مسؤولية الإسلام وإنما هو مسؤولية المسلمين الذين بتلت في سلوكهم - إلى حد كبير - الروح التي كانت سائدة في عهدهم، ذلك على الرغم أن من التاريخ الإسلامي قد عرف بنفس القدر، ازدهاراً للتعدد الثقافي والحضاري والتسامح الديني والفكري وعرف - بدرجات متفاوتة- ظاهرة التعدد السياسي بما يتجاوز عصره بكثير.

لقد أراد الباحث أن يؤكد - عبر فصول كتابه كلها- إن الإسلام إنما جاء لمصلحة البشرية، وأنه يستوعب كل إنجازاتا الخيرة، مثل التقدم العلمي والتنظيم الديمقراطي للشؤون السياسية وضمان حقوق الأفراد 
والشعوب والأقليات والنساء على أساس العدل والمساواة، وإن إقامة دولته الشورية الديمقراطية - دولة الأمة - لا تمثل حاجة للمسلمين فحسب وإنما حاجة للبشرية قاطبة. وهذا الكتاب جدير بالقراءة، للمسلمين خاصتهم وعامتهم، ولغير المسلمين، لأنه يطرح بعض القضايا والإشكاليات الحساسة التي يعالجها الفكر الإسلامي الحديث بكيفية حاسمة على الرغم مما بذل من عطاء فكري واجتهاد ثقافي على طول التاريخ الإسلامي الحديث؛ كل ذلك قد أنجز بمنهجية منظمة وتحليل عميق. ويحمد للمؤلف أنه يقدم استنتاجاته وخلاصاته دون أحكام قطعية أو نظرة أحادية الجانب تلغي فعل العوامل التاريخية والاجتماعية والثقافية والسياسية وغيرها، مما يعكس استشعارا وتقديرا للمسؤولية العلمية. والأمر ذاته يضهر أيضا في التوثيق العلمي والفكري والتاريخي لما أورده من آراء وما تبناه من طروحات، متمثلا في العدد الكبير من المراجع العربية والأجنبية التي استفاد منها المؤلف في كتابه. أما لغة الكتابة فقد جاءت سهلة يسيرة، جزلة الألفاظ ومتينة السبك وجيدة الصياغة. وهي - على كل حال - طابع كتابات الغنوشي

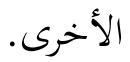

كلمة أخيرة لا بد منها، وهي أن جدارة هذا الكتاب لا تنبع فقط من أهمية موضوعه ولا من خطورة المسائل التي تناولها في الشأن السياسي الإسلامي المعاصر، وإنما أيضا من كونه الحصيلة التي خرج بها مؤلفه من تأمل طويل تم معظمه وهو أسير المعتقل. وينهض هذا الكتاب دليلا على أن مؤلفات المفكرين الحركيين الخالدة جلها قد كتبت في ظروف دخول المحن والخروج منها. وهي دليل إضافي على أن المحنة تخفي في ثناياها منحة مما يعزز مقولة قدوة المجاهدين الصابرين من العلماء، شيخ الإسلام ابن تيمية: "إن نفيي سياحة وسجني خلوة، وقتلي شهادة"، كما أشار إلى ذلك مقدم الكتاب، الدكتور محمد سليم العوا، صاحب المؤلفات السياسية والقانونية المعروفة. 